Gut and Liver, Vol. 11, No. 1, January 2017, pp. 1-2

\title{
Investigation of Factors Affecting Clinical Outcomes after Stent Placement in Malignant Obstruction of the Esophagus or the Gastric Cardia
}

\author{
Ji Bong Jeong and Dong-Won Ahn \\ Department of Internal Medicine, Seoul Metropolitan Government-Seoul National University Boramae Medical Center, Seoul National \\ University College of Medicine, Seoul, Korea
}

\author{
See "Factors That Affect Stent-Related Complications in Patients with Malignant Obstruction of the Esophagus or Gastric \\ Cardia" by Hiroyasu Iwasaki, et al. on page 47, Vol. 11. No. 1, 2017
}

Esophageal cancer is the eighth most common cancer worldwide, and, despite many advances in diagnosis and treatment, the prognosis for esophageal cancer is still poor, with a reported 5-year survival rate ranging from $15 \%$ to $20 \%$. $^{1}$ Over $90 \%$ of patients with esophageal cancer are diagnosed at an advanced stage. $^{2}$ And more than $50 \%$ of the patients with carcinoma of the esophagus or the gastric cardia have inoperable disease at presentation and most of them require palliative treatment to relieve progressive dysphagia. ${ }^{3}$ Various palliative treatments have been attempted for this purpose including rigid plastic intubation, self-expanding metallic stent (SEMS) placement, brachytherapy, external beam radiotherapy, or esophageal bypass surgery. Among these treatments, endoscopic placement of SEMS has become the first-line palliative option for dysphagia and, at present, is commonly used for the palliation of malignant dysphagia. ${ }^{3}$

However, stent-related complications after SEMS placement and recurrent dysphagia resulting from tumor progression or stent migration should be concern in clinical practice. Various stent-related complications such as hemorrhage, perforation, esophago-bronchial fistula, aspiration pneumonia, persistent chest pain, and gastroesophageal reflux have been reported in many studies about SEMS placement in carcinoma of the esophagus or the gastric cardia. ${ }^{1,3-8}$ Considering that these complications may be life-threatening, it would be important to select the patients with high risk of these complications as well as to develop a new favorable type of stent in clinical practice.

To date, studies have reported conflicting results about the relationship between prior radiotherapy or chemotherapy and complications after SEMS placement. ${ }^{1,3-5}$ While, several studies reported that prior radiotherapy and/or chemotherapy increased the risk of life-threating complications after SEMS placement, ${ }^{1,4,5}$ another study showed no significant relationship between prior therapy and stent-related complications. ${ }^{3}$ Regarding the type of stent, studies also reported conflicting results. ${ }^{6-8}$ However, over the course of 25 years, SEMSs have evolved considerably, undergoing numerous modifications, and the therapeutic endoscopist has numerous choices of SEMSs for palliation of dysphagia from carcinoma of the esophagus or the gastric cardia. ${ }^{9}$ Moreover, some types of stent are not commonly available or utilized in some countries. Therefore, at present, further studies with commonly available types of stent are needed to predict the risk according to the types of stent accurately.

In this issue of Gut and Liver, Iwasaki et al. ${ }^{10}$ have attempted a comprehensive approach to investigate the factors affecting stent-related complications in patients with carcinoma of the esophagus or gastric cardia. In their study, data from 53 patients who underwent SEMS placement for malignant obstruction of the esophagus or gastric cardia from single institution were reviewed and multivariate analysis was performed to identify the risk factors of stent-related complications. According to the results of their study, the use of an Ultraflex stent (odds ratio [OR], 19.60) and prior radiation (OR, 25.70) significantly increased the risk of major complications. The authors suggest that higher radial force of the Ultraflex stent be associated with more tissue damage resulting in increased risk of major complications (hemorrhage and perforation) consequently. Regarding prior radiation, the authors mention that the late effects of radiation

Correspondence to: Ji Bong Jeong

Department of Internal Medicine, Seoul Metropolitan Government-Seoul National University Boramae Medical Center, Seoul National University College of Medicine, 20 Boramae-ro 5-gil, Dongjak-gu, Seoul 07061, Korea

Tel: +82-2-870-2222, Fax: +82-2-870-3863, E-mail: jibjeong@snu.ac.kr pISSN 1976-2283 eISSN 2005-1212 https://doi.org/10.5009/gnl16548

@ This is an Open Access article distributed under the terms of the Creative Commons Attribution Non-Commercial License (http://creativecommons.org/licenses/by-nc/4.0) which permits unrestricted non-commercial use, distribution, and reproduction in any medium, provided the original work is properly cited. 
therapy have an important role in the occurrence of major complications.

The risk and characteristics of the stent-related complications can differ depending on the location of obstructive lesion. ${ }^{9}$ In case of obstruction in the proximal esophagus, SEMS placement has been complicated with severe neck and chest pain, at times requiring endoscopic removal of the stent, significant compression of trachea or left/right main stem bronchus with fatal or near-fatal acute hypoxemic respiratory failure, or trachea-esophageal fistula development. ${ }^{9}$ In case of SEMS placement bridging the gastroesophageal junction, by contrast, development of gastroesophageal reflux which results in severe ulcerative esophagitis with hemorrhage as well as aspiration pneumonia, and occurrence of stent migration into the stomach which results in recurrent dysphagia or even bowel obstruction have been serious clinical concerns. ${ }^{9}$ In the report of Iwasaki et al., ${ }^{10}$ location of tumor was upper or middle esophagus in about $58 \%$ of patients, whereas, in $42 \%$ of patients, tumor was located in lower esophagus or gastroesophageal junction. Therefore, it would be needed to perform a subgroup analysis according to the tumor location. Besides, in clinical practice, it is important to achieve persistent improvement of dysphagia after SEMS placement as well as to reduce the SEMS-related complications. Indeed, the risk of recurrent dysphagia after SEMS placement should be also considered as important clinical outcome. In the report of Iwasaki et al., ${ }^{10}$ recurrent dysphagia resulting from tumor overgrowth, tumor ingrowth, stent migration, or food bolus impaction occurred in 12 patients (22.6\%). So, it would be interesting to define the recurrent dysphagia as primary outcome and to perform same analysis to find factors affecting the recurrent dysphagia. The lack of these analyses might be the limitation of study by Iwasaki et al. ${ }^{10}$ Nevertheless, their report should provide clinicians with helpful information for prediction of complications after SEMS placement in malignant obstruction of the esophagus or gastric cardia. And the results of their study might pave the way to perform prospective, multicenter studies about this issue.

\section{CONFLICTS OF INTEREST}

No potential conflict of interest relevant to this article was reported.

\section{REFERENCES}

1. Fuccio L, Scagliarini M, Frazzoni L, Battaglia G. Development of a prediction model of adverse events after stent placement for esophageal cancer. Gastrointest Endosc 2016;83:746-752.

2. Lim H, Kim DH, Jung HY, et al. Clinical significance of early detection of esophageal cancer in patients with head and neck cancer. Gut Liver 2015;9:159-165.

3. Homs MY, Hansen BE, van Blankenstein M, Haringsma J, Kuipers EJ, Siersema PD. Prior radiation and/or chemotherapy has no effect on the outcome of metal stent placement for oesophagogastric carcinoma. Eur J Gastroenterol Hepatol 2004;16:163-170.

4. Kinsman KJ, DeGregorio BT, Katon RM, et al. Prior radiation and chemotherapy increase the risk of life-threatening complications after insertion of metallic stents for esophagogastric malignancy. Gastrointest Endosc 1996;43:196-203.

5. Lecleire S, Di Fiore F, Ben-Soussan E, et al. Prior chemoradiotherapy is associated with a higher life-threatening complication rate after palliative insertion of metal stents in patients with oesophageal cancer. Aliment Pharmacol Ther 2006;23:1693-1702.

6. Siersema PD, Hop WC, van Blankenstein M, et al. A comparison of 3 types of covered metal stents for the palliation of patients with dysphagia caused by esophagogastric carcinoma: a prospective, randomized study. Gastrointest Endosc 2001;54:145-153.

7. May A, Hahn EG, Ell C. Self-expanding metal stents for palliation of malignant obstruction in the upper gastrointestinal tract: comparative assessment of three stent types implemented in 96 implantations. J Clin Gastroenterol 1996;22:261-266.

8. Verschuur EM, Repici A, Kuipers EJ, Steyerberg EW, Siersema PD. New design esophageal stents for the palliation of dysphagia from esophageal or gastric cardia cancer: a randomized trial. Am J Gastroenterol 2008;103:304-312.

9. Weston AP. Stents in the proximal esophagus: tailoring the stent to the patient to achieve success. Gastrointest Endosc 2011;73:1106-1108.

10. Iwasaki H, Mizushima T, Suzuki Y, et al. Factors that affect stentrelated complications in patients with malignant obstruction of the esophagus or gastric cardia. Gut Liver 2017;11:47-54. 